\title{
Zugänge für die Hämodialyse
}

Thalhammer, C ; Segerer, S

DOI: https://doi.org/10.1055/s-0034-1370163

Other titles: Hemodialysis vascular access

Posted at the Zurich Open Repository and Archive, University of Zurich ZORA URL: https://doi.org/10.5167/uzh-101369

Journal Article

Accepted Version

Originally published at:

Thalhammer, C; Segerer, S (2014). Zugänge für die Hämodialyse. Deutsche Medizinische Wochenschrift, 139(31-32):1562-1564.

DOI: https://doi.org/10.1055/s-0034-1370163 


\section{Zugänge für die Hämodialyse}

Christoph Thalhammer ${ }^{1}$, Stephan Segerer ${ }^{2}$

Klinik für Angiologie ${ }^{1}$ und Nephrologie ${ }^{2}$, UniversitätsSpital Zürich, Schweiz

Korrespondenz:

Prof. Dr. med Stephan Segerer

Abteilung für Nephrologie

Universitätsspital Zürich

Rämistasse 100

8091 Zürich, Schweiz

Tel.: $+41(0) 442559698$

FAX: $+41(0) 442554593$

E-mail: Stephan.segerer@usz.ch 


\section{Zusammenfassung}

In den letzten Jahrzehnten sind Anzahl und das Alter der dialysepflichtigen Patienten stark angestiegen. Es handelt sich meist um multimorbide Patienten mit einem besonders hohen kardiovaskulären Risiko. So haben sich die Voraussetzungen für Gefässzugänge für die Hämodialyse deutlich verändert. In vielen Zentren wurden interdisziplinäre Arbeitsgruppen eingerichtet um eine optimale Versorgung der Dialysepatienten zu gewährleisten. Hier diskutieren wir aktuelle Entwicklungen $\mathrm{zu}$ diagnostischen und therapeutischen Aspekten der Hämodialysezugänge. Viele klinische Entscheidungen im Umgang mit Dialysezugängen basieren jedoch immer noch auf persönlichen Erfahrungen und nicht auf guten Studien.

\section{Schlüsselwörter}

Arteriovenöse Fistel, arteriovenöser Graft, Hämodialyse, Peritonealdialyse, Duplexsonographie, Monitoring

\section{Keywords}

Arteriovenous fistula, arteriovenous graft, haemodialysis, peritoneal dialysis, duplex ultrasound, monitoring 


\section{Was ist neu?}

Aufgrund der demographischen Entwicklung sind die Voraussetzungen für native Dialyseshunts schlechter geworden, was sich in einem Trend zu einem höheren Anteil von Dialysekathetern widerspiegelt. Um das Ziel eines funktionierenden Dialyseshunts zum Zeitpunkt urämischer Symptome zu erreichen müssen Patienten optimal vorbereitet werden. Die Duplexsonographie stellt die entsprechenden möglichen Gefässe dar. Die Regel immer möglichst peripher zu beginnen muss an die Situation angepasst werden. So kann es beim älteren Patienten der rasch eine Dialyse benötigt sinnvoll sein einen Shunts am Oberarm anzulegen, als den Versuch zu unternehmen ungünstige Unterarmgefässe zu anastomosieren.

\section{Wann muss ein Nierenfunktionsersatz begonnen werden?}

Ein definitiver Dialysezugang (nativer Dialyseshunt oder eine Kunstoffprothese) sollte verwendbar sein wenn die Dialyse begonnen werden muss. Der richtige Zeitpunkt des Dialysebeginns wurde in einer grossen, randomisierten Studie evaluiert [1]. Über 800 Patienten mit chronischer Niereninsuffizienz wurden zu einem frühen Dialysebeginn (glomeruläre Filtrationsrate zwischen 10 und $14 \mathrm{ml}$ pro Minute) oder einem späten Beginn (5-7 ml/min) randomisiert. Es zeigte sich kein Unterschied in der Gesamtmortalität. Bei Patienten in der letzteren Gruppe wurde die Dialysetherapie knapp 6 Monate später begonnen. Bei etwa 75\% der Patienten wurde die Therapie begonnen bevor sie eine GFR von $7 \mathrm{ml} / \mathrm{min}$ erreicht hatten, da sie Symptome entwickelten. Bei Patienten welche auf die Dialyse vorbereitet wurden kann also mit dem Beginn der Dialyse abgewartet werden bis Symptome der Urämie auftreten. In dieser Studie wurden definitive Dialysezugänge angestrebt um eine Versorgung mit Dialysekathetern zu vermeiden. Aufgrund der demographischen 
Entwicklung nimmt die Zahl der Patienten mit Dialysekathetern jedoch zu. Die Nutzung von Dialysekathetern zu Dialysebeginn ist mit einem erhöhten Risiko an Hospitalisationen, Infektionen und signifikant erhöhter Mortalität im ersten Dialysejahr assoziiert.

Klinische Relevanz: Der richtige Zeitpunkt zum Dialysebeginn beim aufgeklärten Patienten ist beim Auftreten von urämischen Symptomen (der Patient wird diesen im Wesentlichen bestimmen). Zu diesem Zeitpunkt muss ein funktionierender definitiver Dialysezugang vorhanden sein um Katheterkomplikationen zu vermeiden.

\section{Eine frühzeitige Planung ermöglicht dem Patienten die freie Wahl des Dialyseverfahrens und eine optimale Vorbereitung}

Um die Forderung eines definitiven Dialysezuganges bei Dialysebeginn zu erreichen, muss der Patient rechtzeitig aufgeklärt werden. Je nach Verlauf der Nierenerkrankung sollte dies bei einer GFR zwischen 20 und $30 \mathrm{ml} / \mathrm{min}$ thematisiert werden. Nach ausführlicher Aufklärung über die Möglichkeiten (konservative Therapie, Dialyse, Nierentransplantation) wird mit dem Patienten ein Konzept entwickelt. In der Regel besteht dies in der Entscheidung für ein Dialyseverfahren (Peritonealdialyse oder Hämodialyse), parallel erfolgt die Abklärung für die Nierentransplantation. Sollte ein Lebendnierenspender vorhanden sein und die Abklärung rechtzeitig abgeschlossen, so kann ein präemptive Nierenspende angestrebt werden.

Hat sich der Patient für die Peritonealdialyse (PD) entschieden, so sollte etwa 14 Tage vor Dialysebeginn ein PD-Katheter implantiert werden. Die Peritonealdialyse hat besonders am Anfang der Dialysetherapie eine Reihe von Vorteilen so dass viele Zentren (und auch unseres) die Peritonealdialyse als Mittel der ersten Wahl ansehen 
(„PD first"). Insbesondere bei Patienten die keine Shuntmöglichkeit haben, die Hämodialyse also über einen Katheter erfolgen müsste, sollte die Peritonealdialyse gewählt werden, da diese ein besseres Outcome gezeigt hat [8].

Ein nativer Shunt für die Hämodialyse benötigt nach der Operation in der Regel 6-8 Wochen bis dieser benutzt werden kann. Nach Implantation einer Kunststoffprothese kann diese meist bereits nach 2-4 Wochen benutzt werden (je nach Ausmass der perioperativen Schwellung). Aufgrund der immer schlechteren Ausgangslage (insbesondere der schlechten arteriellen Situation) sollte eine Shuntanlage ab einer GFR um $20 \mathrm{ml} / \mathrm{min}$ geplant werden. Je schneller die Nierenfunktion verloren geht und je schlechter die Gefässsituation ist, desto früher. Sobald eine Entscheidung für die Hämodialyse getroffen wurde sollten potentielle Shuntvenen geschont werden und mit systematischer Belastung (z.B. regelmässiges Kneten von Bällen, Hanteln) trainiert werden.

Klinische Relevanz: Eine rechtzeitige Aufklärung des Patienten ermöglicht die freiere Entscheidung des Patienten, ein optimale Vorbereitung und verbessert die Prognose an der Dialyse

\section{Vorbereitung auf die Shuntanlage - Informationen aus dem duplexsonographischen Shuntmapping}

Die duplexsonographische Beurteilung der potentiellen Shuntgefässe hat sich als Bildgebung durchgesetzt, unklar ist jedoch welche strukturellen und funktionellen Gefässeigenschaften die beste Vorhersagekraft haben. Die gereifte radio-cephale Fistel am nicht dominanten Arm entspricht einem "idealen" Gefässzugang. Neben der klinischen Untersuchung gehört die farbkodierte Duplexsonographie zum festen Bestandteil der präoperativen Abklärung vor Shuntanlage um mit der Bestimmung der strukturellen (leider bisher nicht der funktionellen Gefässeigenschaften) eine 
möglichst gute Shuntreifung zur erreichen. Im Vergleich zur alleinigen präoperativen klinischen Untersuchung kann die Rate der erfolgreichen Shuntanlagen durch die Duplexsonographie signifikant verbessert werden (bis zu 97\% der Fälle wurden beschrieben) [4]. Allerdings basieren die Daten zur Wertigkeit der präoperativen Duplexsonographie für die Vorhersage einer Shuntreifung auf wenigen guten Studien und die sonographische Grenzwerte sind immer noch in Diskussion [11]. Empfohlen ist der minimale Durchmesser der Arteria radialis von $2.0 \mathrm{~mm}$ und ein venöser Durchmesser von $2.5 \mathrm{~mm}$ [10]. Kürzlich erschien eine sehr ordentlich durchgeführte, prospektive Beobachtungsstudie an 122 Patienten, die morphologische und funktionelle Parameter auf ihre Vorhersagekraft für die Maturation von radiocephalen Shunts untersuchte [5]. Die inneren Durchmesser der Arteria radialis und der Vena cephalica, die venöse Distensibilität, der Resistance Index und die endotheliale Funktion der Arteria brachialis wurden vor Shuntanlage gemessen. Die besten Prädiktoren für eine Shuntentwicklung waren ein Durchmesser der Arteria radialis von $>1.6 \mathrm{~mm}$, der Vena cephalica von $>1.8 \mathrm{~mm}$ und eine venöse Distensibilität (Änderung des Durchmessers nach venöser Stauung) von $>0.4 \mathrm{~mm}$. Dieser Parameter hatte den grössten Einfluss. Patienten, deren Vene sich um mehr als $0.4 \mathrm{~mm}$ erweiterte, hatten eine 7 -fach höhere Wahrscheinlichkeit einen funktionierende Shunt zu entwickeln [5]. Der Resistance Index unter Hyperämie und die endotheliale Funktion hingegen hatten - entgegen den Daten älterer Studien keinen Einfluss auf die Shuntentwicklung. Die Magnetresonanzangiographie (MRA) ohne Kontrastmittel hat bisher als alternative Untersuchung enttäuscht.

Klinische Relevanz: Die Duplexsonographie vor Anlage erhöht die Wahrscheinlichkeit einer funktionierenden peripheren AV Fistel. Je schwieriger die 
Situation ist (z.B. Adipositas, Voroperationen) desto stärker ist der positive Einfluss der duplexsonographischen Voruntersuchung.

\section{Entscheidung für den "optimalen" Zugang}

Die derzeitigen europäischen Guidelines empfehlen die erste native Fistel so distal wie möglich anzulegen [10]. Je distaler eine Fistel angelegt wird desto grösser ist die Wahrscheinlichkeit, dass diese nicht reift. Daher ist für eine periphere Fistel mehr Zeit bis zu Nutzung zu planen. Native Fisteln haben im Vergleich zu Grafts eine längere Lebensdauer und benötigen weniger Interventionen um diese funktionstüchtig zu halten. Grafts haben den Vorteil schneller benutzbar zu sein. Je jünger und "gesünder" ein Patient ist desto eher sollten alle peripheren Möglichkeiten ausgeschöpft werden. Ob dies bei unseren älteren, multimorbiden Patienten richtig ist wurde inzwischen in mehreren Studien untersucht. Bei Diabetikern konnte gezeigt werden, dass der Nachweis von Verkalkungen in der Arteria radialis mit einer signifikant schlechteren primären und sekundären Durchgängigkeit assoziiert [3]. In einer retrospektiven Analyse von älteren Patienten wurde eine periphere radio-cephale Fistel mit einer Oberarmfistel (welche eine Perforansvene in der Ellbeuge benutzte) verglichen (jeweils etwa 50 Patienten in beiden Gruppen). Die Shunts welche in der Ellenbeuge angelegt wurden hatten eine signifikant bessere primäre und sekundäre Offenheitsrate nach 24 Monaten $(78.2 \%$ vs $62 \%, P=0.04)[7]$.

Im direkten Vergleich zwischen einer nativen brachio-basilären Fistel und einem Graft zeigte sich für die native Fistel eine längere Hospitalisation und eine längere Zeit bis die native Fistel benutzt werden konnte. Grafts hatten eine höhere Rate später Komplikationen mit notwendigen Interventionen [6]. Primäre und sekundäre Offenheitsraten waren gleich. 
Daher kann die klinische Entscheidung letztlich davon abhängig gemacht werden wie rasch die Fistel gebraucht wird und wie lange die Lebenserwartung des Patienten ist. Das Alter des Patienten ist hierbei nicht von Bedeutung, da die Zahl der funktionierenden Fisteln bei Patienten über und unter 65 Jahren nicht unterschiedlich waren [9].

Klinische Relevanz: Die Regel je peripherer desto besser muss an die klinische Situation angepasst werden [2]. Je multimorbider ein Patient ist und je schneller der Shunt gebraucht wird, desto geringer ist die Chance dass er von einer peripheren nativen Fistel einen Vorteil hat.

Literatur:

1. Cooper BA, Branley $\mathrm{P}$, Bulfone $\mathrm{L}$, et al. A randomized, controlled trial of early versus late initiation of dialysis. N Engl J Med 2010; 363: 609-619

2. Drew DA, Lok CE. Strategies for planning the optimal dialysis access for an individual patient. Curr Opin Nephrol Hypertens 2014, DOI: 10.1097/01.mnh.0000444815.49755.d9

3. Georgiadis GS, Georgakarakos El, Antoniou GA, et al. Correlation of preexisting radial artery macrocalcifications with late patency of primary radiocephalic fistulas in diabetic hemodialysis patients. J Vasc Surg 2014, DOI: 10.1016/j.jvs.2014.02.042

4. Ilhan G, Esi E, Bozok S, et al. The clinical utility of vascular mapping with Doppler ultrasound prior to arteriovenous fistula construction for hemodialysis access. J Vasc Access 2013; 14: 83-88

5. Jemcov TK. Morphologic and functional vessels characteristics assessed by ultrasonography for prediction of radiocephalic fistula maturation. J Vasc Access 2013; 14: 356-363

6. Morosetti M, Cipriani S, Dominijanni S, et al. Basilic vein transposition versus biosynthetic prosthesis as vascular access for hemodialysis. J Vasc Surg 2011; 54: 1713-1719

7. Palmes $D$, Kebschull $L$, Schaefer RM, et al. Perforating vein fistula is superior to forearm fistula in elderly haemodialysis patients with diabetes and arterial hypertension. Nephrol Dial Transplant 2011; 26: 3309-3314

8. Perl J, Wald R, McFarlane $\mathrm{P}$, et al. Hemodialysis vascular access modifies the association between dialysis modality and survival. J Am Soc Nephrol 2011; 22: $1113-1121$ 
9. Renaud CJ, Pei JH, Lee EJ, et al. Comparative outcomes of primary autogenous fistulas in elderly, multiethnic Asian hemodialysis patients. J Vasc Surg 2012; 56: 433-439

10. Tordoir J, Canaud B, Haage P, et al. EBPG on Vascular Access. Nephrol Dial Transplant 2007; 22 Suppl 2: ii88-117

11. Wong CS, McNicholas N, Healy D, et al. A systematic review of preoperative duplex ultrasonography and arteriovenous fistula formation. J Vasc Surg 2013; 57: 1129-1133 